\title{
Assessment of Commodity Classification for Sprinkler Protection Using Representative Fuels
}

\author{
YIBING XIN and FRANCESCO TAMANINI \\ FM Global \\ 1151 Boston-Providence Turnpike \\ Norwood, MA 02062 USA
}

\begin{abstract}
In order to assess the classification of commodities for sprinkler protection, fire suppression tests were conducted using representative fuels. The measured heat release rates and applied water fluxes are used to calculate the critical delivered flux (CDF) and develop a suppression correlation for each tested commodity. The heat release rate (HRR) measurements together with the CDF calculations show that the $\mathrm{CDF}$ can be obtained within an uncertainty of $\pm 3 \mathrm{~mm} / \mathrm{min}$. The CDF values are then combined with the measured convective HRR to project the critical sprinkler discharge flux (SDF) - the minimum water flux above which the fire will be controlled. Comparison of SDF values and full-scale sprinkler test results shows that the SDF is a reasonable choice for ranking commodities. Examination of the calculated CDF values using the suppression correlations suggests that the ranking remains the same for various fuel array heights. Furthermore, the responses to suppression by the exposed plastics are very different from those of the cartoned fuels, indicating that these two types of commodities require different treatment.
\end{abstract}

KEYWORDS: commodity classification, sprinkler protection, suppression, fire growth.

\section{NOMENCLATURE LISTING}

$\begin{array}{llll}\dot{m}^{\prime \prime} & \text { water flux (mm/min) } & \text { ex } & \text { extinguishment } \\ n & \text { number of tiers in a fuel array } & f & \text { final } \\ \dot{Q} & \text { heat release rate }(\mathrm{kW}) & g & \text { growth } \\ T_{\text {link }} & \text { sprinkler link temperature }\left({ }^{\circ} \mathrm{C}\right) & \max & \text { maximum } \\ \dot{Q}_{w} & \text { water cooling rate }(\mathrm{kW}) & \mathrm{s} & \text { suppression } \\ \dot{Q}_{w}^{*} & \text { dimensionless cooling power } & \text { abbreviations } \\ \Delta Q & \text { energy release }(\mathrm{kJ}) & \text { ADD } & \text { actual delivered density } \\ t & \text { time (s) } & \text { CDF } & \text { critical delivered flux } \\ \text { subscripts } & \text { DS } & \text { FM Global data sheet } \\ 0 & \text { freeburn } & \text { HRR } & \text { heat release rate } \\ b e & \text { beginning of extinguishment } & \text { SDF } & \text { sprinkler discharge flux } \\ \text { conv } & \text { convective } & \text { SPC } & \text { standard plastic commodity } \\ \text { const } & \text { constant } & \text { WAA } & \text { water application apparatus }\end{array}$

\section{INTRODUCTION}

Combustible commodities are stored in various geometries in industrial and commercial facilities. The central issue for fire protection research is how to define the appropriate protection for a given commodity. This is a very challenging problem since the combinations of material properties and storage geometries are almost unlimited, but only a few representative fuels can be studied systematically. Therefore, a methodology is needed to link the limited research results to the unlimited commodities in the real world. Commodity classification is such a methodology by which commodities are categorized into different classes according to material properties and fuel geometries for fire protection purposes. Once a commodity is classified, the protection recommendations of the representative fuel for that class will be assigned to the commodity of interest. In essence, commodity classification is a mapping scheme that connects the protection recommendations of representative fuels to the commodities in the real world.

Commodity classification was introduced based on full-scale sprinkler test results in the late 1970s. In 1990, Chicarello and Troup [1] developed the first experimental classification method, where fire suppression tests were conducted using two-pallet-load long, two-pallet-load deep and two-tier high 
$(2 \times 2 \times 2)$ fuel arrays and the measured HRRs were introduced into empirical correlations to calculate the commodity ranking. In addition, $\mathrm{Wu}$ [2] attempted to develop a new commodity classification method by the use of flame heat flux measurements. However, the cost of full-scale sprinkler tests is high and only limited full-scale data for standard commodities are available for comparison; the empirical correlations used by Chicarello and Troup [1] can be hardly extrapolated to new commodities; and the measured flame heat fluxes by $\mathrm{Wu}$ [2] showed no correlations with the fire hazards of well known commodities. Consequently, it remains questionable how to evaluate the fire hazards of commodities and whether existing classes can be regrouped or simplified. As a first step in addressing these questions, this work focuses on the development of a technical approach to assess commodity classification for sprinkler protection using representative fuels.

\section{TECHNICAL APPROACH}

The technical approach is developed from a conceptual analysis of fire growth and suppression processes. (Fig. 1.) The key physics in rack storage fire growth is flame heat transfer. For vertical fire spread, radiation is the dominant heat transfer mode; while for lateral spread, it is the combination of radiation and convection. When the fire grows to a certain size, ceiling sprinklers will open to discharge a preset water flow. In the present work, the water flow rate per unit area covered by the sprinkler is defined as Sprinkler Discharged Flux (SDF). For sprinklers to work effectively, the water spray has to penetrate the fire plume to reach the burning surface or wet the unburned fuel at the periphery. The water flux surviving in the penetration process is defined as Actual Delivered Density (ADD). Chan et al. [3] conducted an experimental study to measure ADD under various ceiling clearances and HRRs. The results show that the major variables affecting ADD are the convective HRR, the ceiling clearance and spray properties such as droplet diameter and spray momentum. This work provides the basis to link the SDF and ADD under a broad range of conditions and, in particular, for that corresponding to when the fire is barely controlled.

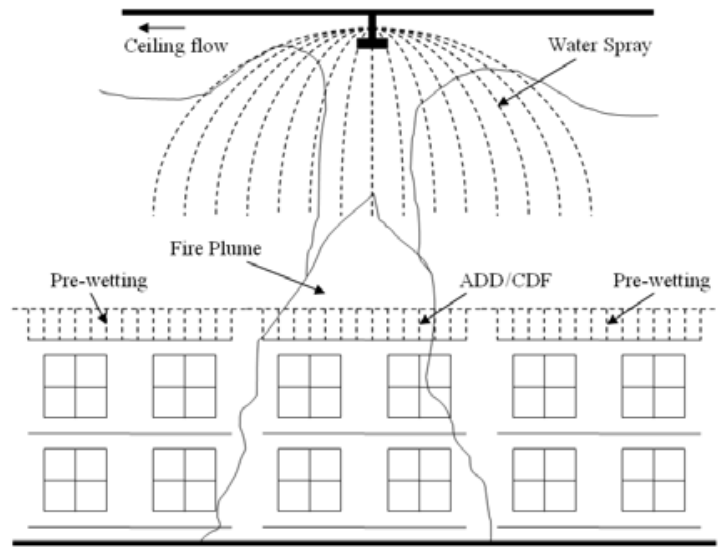

Fig. 1. Schematic of fire growth and suppression.

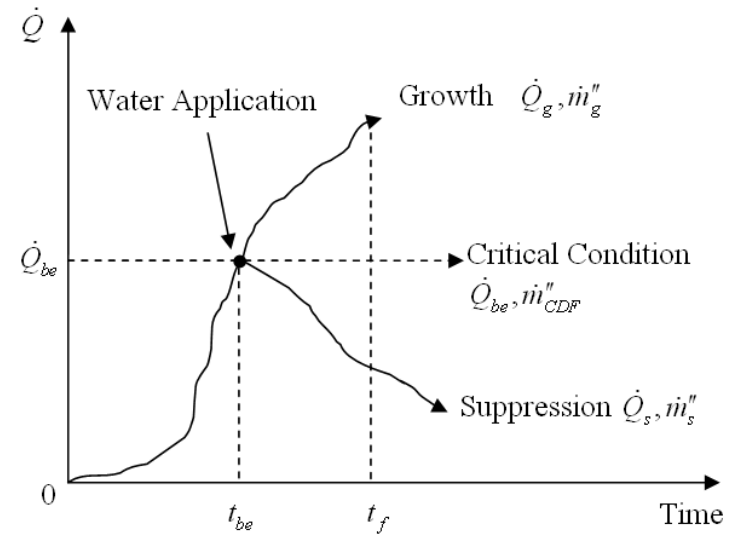

Fig. 2. Illustration of the CDF concept.

The water flux applied on top of the fuel array is crucial to characterize the response of the commodity to suppression. The physics of suppression may include flame extinction in gas phase by evaporative cooling and oxygen displacement, and extinction of solid burning surface by direct droplet impingement and water cascading along solid fuel surface. Due to the strong momentum and intense volumetric HRR of rack storage fires, as well as the shielding effect of the solid commodities, it is assumed that the dominant extinguishment mechanism for burning surface is water cascading along the solid commodity. Although the details of this mechanism are not currently well understood, the water flux delivered to the top of the fuel array has to be sufficient to prevent further fire growth under this mechanism. The minimum value of this water flux is defined as the Critical Delivered Flux (CDF) in this work. Based on this analysis, the ADD has to be equal or greater than the CDF to prevent fire growth. If the CDF is measured for a given commodity and a correlation between ADD and SDF is established, the protection recommendations in terms of SDF can be projected. Since the SDF is the key variable that determines the level of fire hazard for sprinkler protection, the SDF under critical conditions should be used to rank commodities for protection purposes. This is the technical approach that will be followed in the present work. 
Clearly, this technical approach requires the determination of CDF and the development of a correlation of ADD and SDF. Furthermore, a generalized approach is needed to examine the SDF-based ranking under various conditions. This is accomplished by the development of a suppression correlation of fire damage as a function of water cooling power, fire burning intensity and other relevant fuel properties.

Note that in the present work, the term flux is used in CDF and SDF, instead of density as in ADD and required delivered density, in order to emphasize that these quantities are volumetric water flow rates per unit area, and to differentiate them from the concepts of ADD and required delivered density, which have been traditionally associated with the development of early-suppression fast-response sprinklers.

\section{Determination of Critical Delivered Flux}

The CDF is determined based on the mathematical description of the concept illustrated in Fig. 2. After an initial freeburn period, the sprinkler actuation condition will be satisfied and the water be applied. This particular moment is the beginning of extinguishment $t_{b e}$ and the corresponding chemical HRR is $\dot{Q}_{b e}$. Thereafter, the fire may continue to grow with fire size $\dot{Q}=\dot{Q}_{g}$ if the applied water flux $\dot{m}^{\prime \prime}=\dot{m}_{g}^{\prime \prime}$ is insufficient, or be suppressed with $\dot{Q}=\dot{Q}_{s}$ under sufficient water flux $\dot{m}^{\prime \prime}=\dot{m}_{s}^{\prime \prime}$. Conceptually, there is a critical water flux, i.e. CDF, upon which the fire is barely controlled and the corresponding HRR curve levels off for a significant period of time before the fuel burns out. This CDF, although nearly impossible to realize experimentally, is the key variable to characterize the minimum level of successful suppression. The $\mathrm{CDF}$ can be approximated to a first order by the use of an integrated HRR, since the impact of the delivered water flux is characterized by the HRR in a period of time of $t>t_{b e}$. Assuming that the water flux in a controlled and an out-of-control test are $\dot{m}_{s}^{\prime \prime}$ and $\dot{m}_{g}^{\prime \prime}$, respectively, then the CDF can be calculated by linear interpolation as

$\left(\Delta Q_{g}-\Delta Q_{b e}\right) /\left(\Delta Q_{b e}-\Delta Q_{s}\right)=\left(\dot{m}_{g}^{\prime \prime}-\dot{m}_{b e}^{\prime \prime}\right) /\left(\dot{m}_{C D F}^{\prime \prime}-\dot{m}_{s}^{\prime \prime}\right)$,

where $\Delta Q_{s}=\int_{t_{b e}}^{t_{f}} \dot{Q}_{s} d t, \Delta Q_{g}=\int_{t_{b e}}^{t_{f}} \dot{Q}_{g} d t$ and $\Delta Q_{b e}=\left(t_{f}-t_{b e}\right) \dot{Q}_{b e}$ are the total energy release, and $\dot{m}_{C D F}^{\prime \prime}$ is the CDF. The beginning time $t_{b e}$ is determined by the sprinkler activation, which is calculated on the fly in a fire test using the measured convective HRR for a given sprinkler condition including link temperature, response time index and ceiling clearance. The ending time $t_{f}$ is often determined by the capacity of the test apparatus when the fire is out of control. Note that all quantities in Eq. 1 are either measured or known, except $\dot{m}_{C D F}^{\prime \prime}$ which is calculated with an uncertainty between $\left(\dot{m}_{C D F}^{\prime \prime}-\dot{m}_{s}^{\prime \prime}\right)$ and $\left(\dot{m}_{g}^{\prime \prime}-\dot{m}_{C D F}^{\prime \prime}\right)$. It is recognized that the above approximation is sensitive to the selection of the final time $t_{f}$. However, examination of test data shows that the sensitivity is small, as long as the suppression time $\left(t_{f}-t_{b e}\right)$ is sufficiently long, a condition which can be reasonably set as $t_{f}-t_{b e} \gg t_{b e}$.

\section{ADD Correlation}

The ADD correlation connects the CDF and the SDF. For a given CDF value, the SDF has to be large enough to produce an ADD greater than or equal to the CDF. Therefore the question is how to project the SDF under the critical condition of $\mathrm{ADD}=\mathrm{CDF}$ with the measured HRR at the beginning of extinguishment. This question can be answered by resorting to the work of Chan et al. [3], who measured the ADDs under various SDFs and convective HRRs for six upright spray automatic sprinklers. All the measurements are qualitatively similar and one example is shown in Fig. 3. for a $3.05 \mathrm{~m}$ ceiling.

It is clear from Fig. 3. that the ADD values are a function of SDF and convective HRR for a given ceiling clearance. For all the tested sprinklers, the ADD values are equal to the SDF when the convective HRR vanishes, and decrease with increasing $\dot{Q}_{\text {conv }}$ and decreasing SDF. When the convective HRR increases by the same amount, the ADD tends to drop slightly more for larger SDF, suggesting that the normalized ADD is affected by the value of SDF. Another interesting observation is that the ADD appears to decrease only 
to a certainly level when the convective HRR is sufficiently large, e.g. > 1.5 MW. For a $3.05 \mathrm{~m}$ ceiling, the visible flame height is equal to or greater than the ceiling clearance when the HRR is equal to or greater than 1.5 MW. Under this condition, the water discharged at ceiling level has to penetrate through the visible flame zone, where the gas velocity is accelerated due to buoyancy and the mean temperature is approximately a constant of $1300 \mathrm{~K}$ regardless of the fire size. Since the buoyancy is largely determined by the gas temperature and the ceiling clearance, both plume temperatures and momentum are not sensitive to the fire size as along as it is sufficiently large. As a result, a certain amount of water can always penetrate through the fire plume. This is clearly shown in Fig. 3. and by the rest of the data in Ref. [3] An immediate implication of this observation is that the ADD correlation developed based on such a data set can be applied to larger convective HRRs with reasonable confidence.

A quantitative relationship between SDF, ADD and $\dot{Q}_{\text {conv }}$ can be described by the following empirical expression

$m^{*}=\dot{m}_{C D F}^{\prime \prime} / \dot{m}_{S D F}^{\prime \prime}=1-k_{1}\left[1-\exp \left(-\dot{Q}_{\text {conv }} / \dot{Q}_{c o n s t}\right)\right]\left(\dot{m}_{S D F}^{\prime \prime}\right)^{k_{2}}$,

where $\dot{Q}_{\text {const }}$ is a constant of $1116 \mathrm{~kW}$, and $k_{1}=0.171$ and $k_{2}=0.327$ are correlation coefficients. The core function of Eq.2 is the exponential decay of normalized ADD $\left(m^{*}=\dot{m}_{C D F}^{\prime \prime} / \dot{m}_{S D F}^{\prime \prime}\right)$ with $\dot{Q}_{\text {conv }}$, which is adjusted by a power function of SDF. The ADD is set to be equal to the SDF in the absence of fire. Note that the ceiling clearance is not included in Eq. 2 as a variable because this correlation is developed only for a fixed ceiling clearance of $3.05 \mathrm{~m}$ for the present work. Inclusion of the ceiling clearance into this type of correlation can certainly be accomplished by further study of the experimental data in Ref. [3].

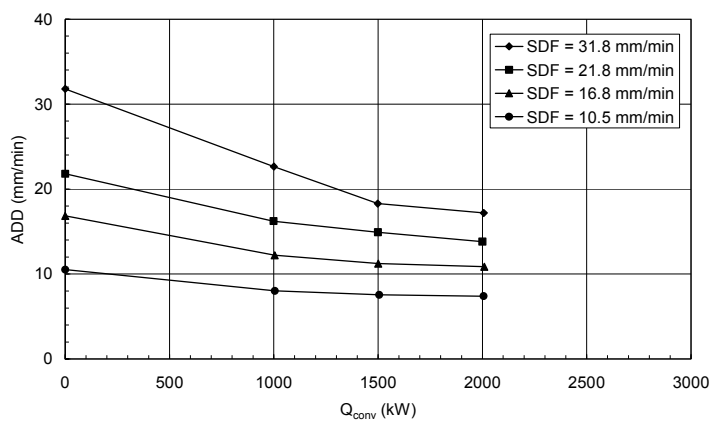

Fig. 3. ADD as a function of SDF and $\dot{Q}_{\text {conv }}$.

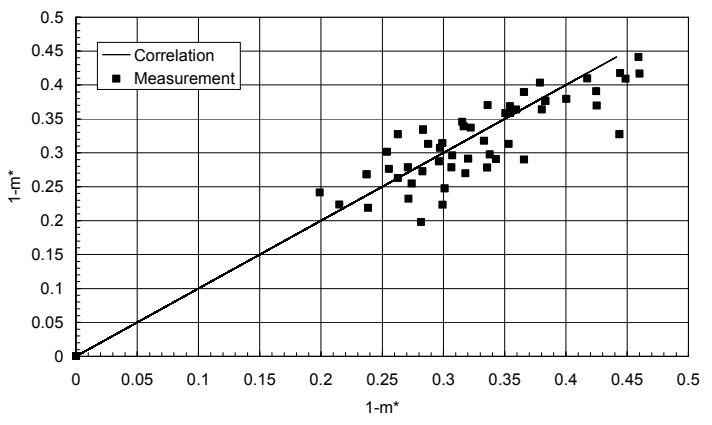

Fig. 4. ADD correlations and measurements.

From Eq. 2. the SDF value can be solved implicitly for a given set of CDF and convective HRR at the beginning of extinguishment. The final ranking of commodities should be based on the SDF, which closely simulates the results of a full-scale sprinkler test. However, the critical SDF values are often obtained only for a specific sprinkler condition because the CDF measurements are expensive. In order to examine the validity of the SDF-based ranking at various conditions, e.g. a higher fuel array, it is necessary to develop a generalized correlation for the effectiveness of fire suppression as a function of controlling variables including the fire size at the beginning of extinguishment and the applied water flux.

\section{Suppression Correlation}

The suppression correlation is developed from a qualitative understanding of the controlling physics. When water is applied on top of the fuel in a rack storage fire, both continuous films and discrete rivulets can be generated to cascade on the vertical fuel surfaces. The cooling and wetting of cascading water competes with the heating by flame radiation and convection. The end result depends on the relative strength of water cooling and wetting as well as flame heat transfer to the fuel. The problem is complex due to the multiphysics involved, among which flame heat transfer and surface extinguishment are yet to be quantified with confidence. Therefore, the effectiveness of suppression is correlated with the controlling variables as

$\Delta Q_{e x} / \Delta Q_{0}=a\left(\dot{Q}_{w} / \dot{Q}_{b e}\right)^{b}\left(\dot{Q}_{b e} / \dot{Q}_{\max }\right)^{c} n^{e}$, 
where $\Delta Q_{e x}$ is the total heat released during fire extinguishment, $\Delta Q_{0}$ is the same quantity in a freeburn test, $\dot{Q}_{w}$ is the water cooling rate defined as the product of water discharge flow rate and the heat of vaporization, $\dot{Q}_{b e}$ is the HRR at the beginning extinguishment, $\dot{Q}_{\max }$ is the maximum HRR in a freeburn test, $n$ is the number of tiers of the fuel array and $a, b, c$ and $e$ are correlation coefficients that need to be determined by experimental data. The quantity $\Delta Q_{0}$ is estimated from the mass and the heat of combustion of the fuel components, although the result is likely greater than that in a real freeburn fire; the measurement of $\dot{Q}_{\max }$ is limited by the calorimeter capacity. Consequently, an estimate is made for $\dot{Q}_{\max }$ using data from smaller fuel array. Although the relative cooling power $\dot{Q}_{w} / \dot{Q}_{b e}$ and burning intensity $\dot{Q}_{b e} / \dot{Q}_{\max }$ as well as storage height $n$ qualitatively determine the effectiveness of fire suppression denoted by the damage fraction $\Delta Q_{e x} / \Delta Q_{0}$, the functional form of Eq. 3 is completely empirical. The existence of this type of correlation is supported by experimental data of Yu et al. [4] and Chicarello and Troup [1] shown in Fig. 5. From the comparison, the damage fraction appears to correlate with the controlling variables with some degree of uncertainty.
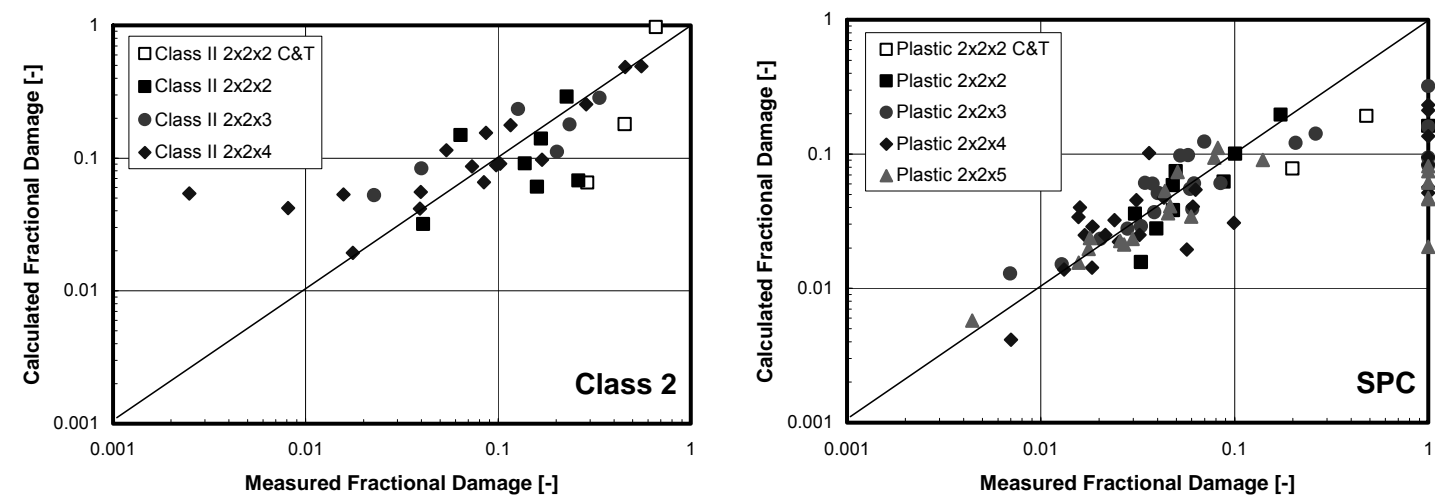

Fig. 5. Suppression correlations based on data from previous work.

It should also be pointed out that from the analysis of previous data [1,4], it appears that there is a relationship among the correlations coefficients: $c-e-b=1$ with $\pm 15 \%$ of uncertainty. This relationship implies that the water cooling rate $\dot{Q}_{w}$ is approximately proportional to the fuel array height in terms of the number of tiers $n$, which is also confirmed by the analysis of Yu et al. [4] In the rest of the present work, therefore, it is assumed that the correlation coefficient $e$ associated with the variation of fuel array height can be determined from the aforementioned relationship.

The development of the correlation for a given fuel requires at least four additional suppression tests with various HRRs and water fluxes in addition to those needed for the CDF measurements. Despite the extra cost, the development of suppression correlations is deemed necessary to examine the SDF-based ranking discussed earlier, under various conditions such as higher fuel arrays. The variability of SDF-based ranking is the key to establishing the range of applications for the commodity classification.

\section{EXPERIMENTAL METHOD}

Fifty-six fire suppression tests were conducted using a Water Application Apparatus (WAA) to measure the CDF and develop suppression correlations for representative commodities. The experimental method is designed to integrate the fuel array and the WAA with a special procedure to achieve accurate control of water application.

\section{Commodities}

In existing national standard [5] and FM Global Loss Prevention Data Sheets (DS) [6], commodity classes include Class $1-4$, cartoned expanded and unexpanded plastic, and exposed expanded and unexpanded 
plastic commodities. As described in Ref. [6], each class covers a range of combinations of combustible materials and storage geometries. In order to study the protection recommendations for each class, a representative fuel has to be selected. Historically [1], Class 1 commodity has been represented by glass jars stored in single-wall corrugated boxes; Class 2 by metal liners in tri-wall corrugated boxes; Class 3 by paper cups in single-wall corrugated boxes; cartoned unexpanded plastic commodity, also known as Standard Plastic Commodity (SPC), by polystyrene cups in single-wall corrugated boxes; cartoned expanded plastic commodity by polystyrene meat trays in single-wall corrugated boxes; exposed unexpanded plastic commodity by plastic pallets made of high-density polyethylene; and exposed expanded plastic commodity by polystyrene meat trays wrapped in plastic bags. These commodity classes and the corresponding representative fuels have been established mainly for evaluating the fire hazard for sprinkler protection in rack storage, which comprises a large portion of commodities in industrial and commercial facilities. Therefore, rack storage of Class 2, 3, 4, cartoned unexpanded and expanded plastic, and exposed unexpanded and expanded plastic commodities are used in the present work. Each pallet load of these representative commodities is placed on a wood pallet consistent with industry practice. More detailed descriptions of these commodities can be found in Ref. [1] and [2].

In contrast to the $2 \times 2 \times 2$ fuel array used in previous work [1, 2], a $2 \times 4$ and 3-tier high fuel array is used in the present study. A schematic of the test setup is shown in Fig. 6. The increased fuel array height allows the fire development to better approximate that of full-scale tests and the added target stacks allow the observation of lateral fire spread. This test setup, although significantly more expensive than that in previous work, is believed to be an essential requirement for a proper simulation of the fire growth and suppression processes of full-scale conditions.

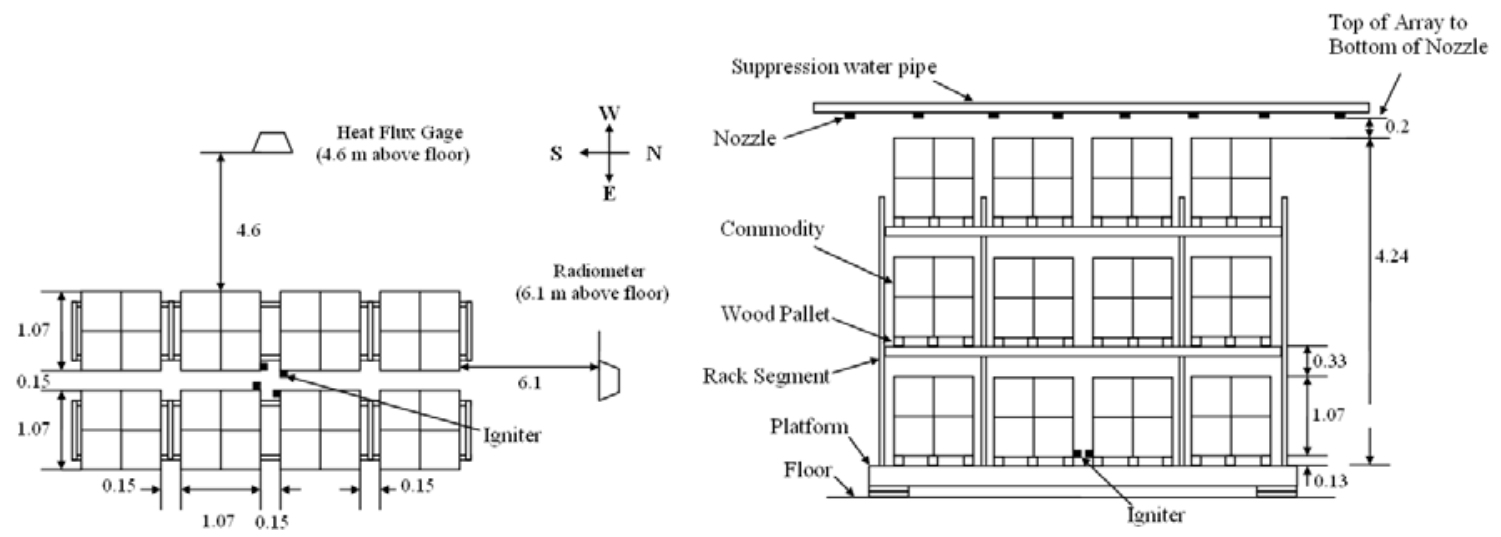

Fig. 6. Schematic of test setup: plan view (left) and elevation (right) view (units in meters).

\section{Test Apparatus}

Once the fuel array is ignited and the fire grows to the predetermined condition, a uniform water flux is applied on top of the fuel by the use of the WAA. The WAA consists of a water supply system, a bypass loop and a water applicator, located below a 20 MW calorimeter. Water is supplied from the laboratory main water outlet, filtered and regulated before entering a solenoid valve connected to a process controller. The process controller constantly compares the set value and the measured flow rate and adjusts the solenoid valve to achieve the prescribed flow rate. Since the water is applied after a period of freeburn, a bypass loop is used to allow the water to flow as close to the water applicator as possible before being switched into the applicator. The initial water flow rate and pressure in the bypass loop is carefully selected to minimize the delivery time, which is delayed mainly by purging the air in the suppression pipes. Usually a high flow rate of $379-568 \mathrm{~L} / \mathrm{min}$ is used for $2-5$ seconds according to the predetermined condition to purge the air in the water applicator. Once the air is purged, the water flow rate is reduced to the preset value either automatically or manually, and kept constant till the end of the test. The applied water fluxes are between $0.41-4.1 \mathrm{~mm} / \mathrm{min}$ and operating pressures are $60-207 \mathrm{kPa}$. Under these conditions, each nozzle located $0.2 \mathrm{~m}$ above the fuel can create a uniformly covered area of $0.61 \mathrm{~m} \times 0.61 \mathrm{~m}$. To achieve uniform water fluxes, different full-cone nozzles are selected so that a mean water droplet diameter of 1.75 $\mathrm{mm}$ with $20 \%$ standard deviation is maintained approximately in all the tests. Since the dominant 
suppression mechanism is water cascading along solid surface, the variation of droplet diameters is considered acceptable.

\section{Test Procedures and Conditions}

A typical test starts from ignition at the center flue as shown in Fig. 6. The fire then undergoes a freeburn period until a predetermined condition is reached. At this moment, the water flow is switched from the bypass loop to the suppression pipes to purge the air and then promptly reduced to a preset value. The test will be terminated if there is no further development of the fire, or the fire size grows to the calorimeter limit. Clearly the key to a successful test is the accurate control of the water application. To achieve this, the HRR measurement requires careful treatment.

First of all, the ambient background on temperature and gas concentration measurements is eliminated by subtracting pre-test data averaged over 120 seconds. Then, a digital channel for ignition is used as a multiplier to the convective HRR to avoid sprinkler link temperature drifting before ignition. The effect of the delay on HRR measurements is compensated by a first order projection of the sprinkler link temperature increase with respect to time. Examination of test data indicates that this projection scheme reduces about $80 \%$ of the total time delay. In addition, the unphysical spikes in the mass flow due to pressure measurements are eliminated by relaxing the mass flow rate over a period of 10 seconds, and using the relaxed mass flow in the convective HRR calculations. Although the relaxation causes a delay in the mass flow measurement, the impact of the delay is deemed acceptable for the present work due to the relatively constant mass flow maintained during the test.

Two types of conditions are used to control the water application: a set of sprinkler actuation conditions, and a fixed convective HRR. The sprinkler actuation conditions are selected for a hypothetical sprinkler with a link temperature of $141^{\circ} \mathrm{C}$ and a response time index of $276(\mathrm{~m} \cdot \mathrm{s})^{1 / 2}$, located $3.05 \mathrm{~m}$ above the top surface of the fuel array. The link temperature of this hypothetical sprinkler is calculated on the fly in the tests using the ceiling flow correlations developed by Kung et al. [7], which are only applicable to the scenarios where visible flame heights are equal to or less than the ceiling clearance. In order to avoid extrapolating the correlations to the scenario with flames extending on the ceiling level, the hypothetical sprinkler is placed along the fire plume axis. In addition to the sprinkler conditions, fixed convective HRRs are also used to control water application for the development of the suppression correlations.

For all the tests using corrugated cardboard commodities, the moisture content is controlled between $4-8$ \% (by weight) by drying the central fuel stacks adjacent to the ignition location in a kiln. The water application conditions are either the sprinkler actuation or a predetermined HRR. The HRR value is prescribed by the convective, instead of chemical HRR, because the former is less delayed and thus easier to control in the test. For the determination of CDF values, suppression tests were carried out with various water fluxes until the critical values could be narrowed within $\pm 3 \mathrm{~mm} / \mathrm{min}$.

\section{RESULTS AND DISCUSSIONS}

\section{Critical Delivered Flux}

The first step to obtain CDF is to distinguish a successfully controlled fire test from an out-of-control case based on qualitative judgment. For the test setup used in this work, a fire is considered out of control when the visible flames spread laterally to one end of the fuel array (4-pallet-load long). It has been observed in the tests that the fire propagation can bias to one lateral direction, but the entire fuel array is always inevitably engulfed in the fire as long as the propagation reaches one end. This qualitative observation is of particular importance to fast propagating fires such as those fueled by exposed meat trays. In these tests, the central stacks adjacent to the ignition location may be all consumed, but the edge stacks can survive if a sufficient water flux is applied. Therefore, qualitative judgment on whether the fire is controlled is made before calculating the CDF values using the HRR measurements.

Two examples of the measured HRRs are shown in Fig. 7. The two HRR curves used in the calculation are those with 12.2 and $15.5 \mathrm{~mm} / \mathrm{min}$ for the SPC and 12.2 and $14.2 \mathrm{~mm} / \mathrm{min}$ for the plastic pallets. In the two tests for the same commodity, the beginning time of extinguishment $t_{b e}$ may vary slightly. Therefore, the actual value of $t_{b e}$ for each test is used in the CDF calculation, while the final time of extinguishment $t_{f}$ is 
determined by the case where the fire is out of control as discussed earlier. The sensitivity of calculated CDF values to $t_{f}$ was examined for Class 2 and 3 and SPC by reducing $t_{f}$ to different values. The results showed that the impact on the calculated CDF values is not significant as long as the final time $t_{f}$ is sufficiently long compared to $t_{b e}$.
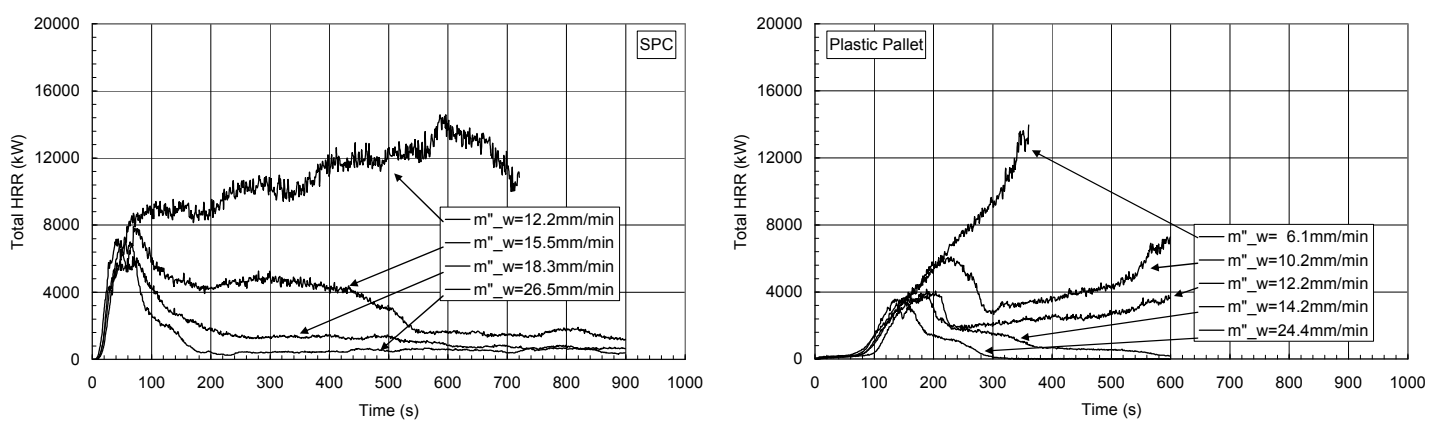

Fig. 7. Total HRR measurements for SPC and plastic pallets.

Comparison of CDF values for the representative commodities is shown in Fig. 8., together with the measured HRRs at the beginning of extinguishment, $\dot{Q}_{b e}$. In terms of critical water fluxes applied directly on top of the fuel array to control the fire, Class 2 presents the lowest requirement while the exposed meat trays require the most; the differences between Class 3, Class 4 and plastic pallet commodities are within the uncertainty range of the measurements, hence these commodities essentially require the same amount of water; the cases of SPC and cartoned meat trays are also similar. These observations, although interesting, have to be combined with the measured convective HRRs at the beginning of extinguishment to calculate the SDF values for each commodity, as discussed in previous section on ADD correlation.
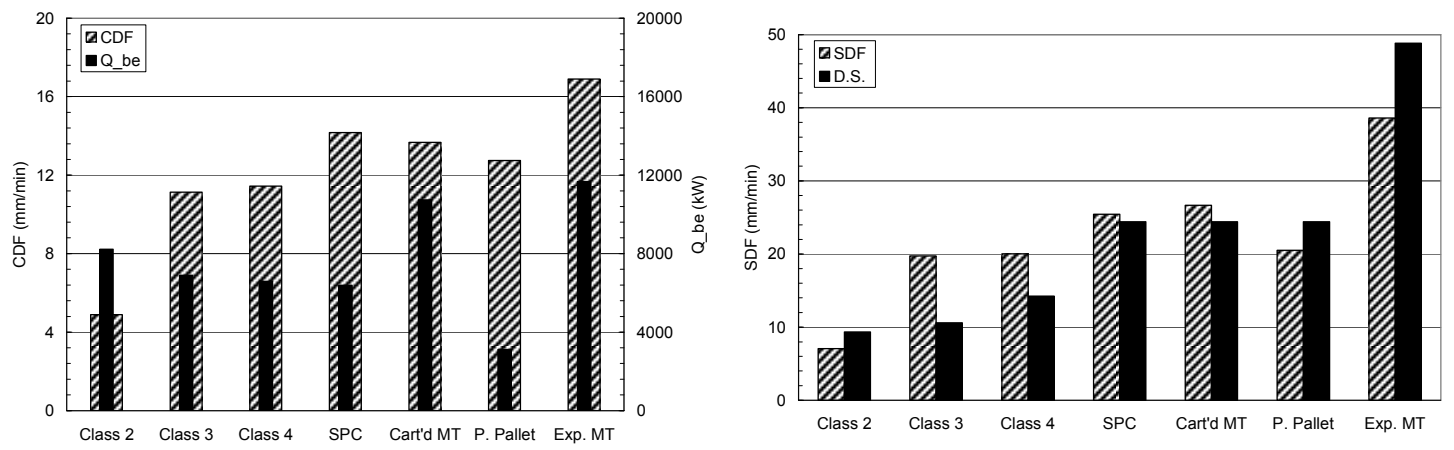

Fig. 8. Calculated CDF and measured $\dot{Q}_{b e}$.

Fig. 9. SDF and protection recommendations in the DS

\section{Sprinkler Discharge Flux}

The SDF values are calculated by solving Eq.2 using the measured CDFs and convective HRRs from the fire tests. The results are compared to the protection recommendations in the DS [6] (Fig. 9.) From the comparison, the rankings based on the SDF and the protection recommendations are consistent for all the commodities if the uncertainties associated with the SDF values are considered. However, there are relatively large differences between the SDF values and the DS recommendations for Class 3 and Class 4 commodities. Since the protection recommendations for these two commodities have been developed on the basis of very limited full-scale sprinkler test data, the relatively large differences do not necessarily invalidate the technical approach used in the present work. A possible reason for the discrepancies is that the protection recommendations for Class 3 commodities have been developed for densely packed cellulosic commodities such as paper plates stored in cardboard boxes for the food industry, which is deemed to be less hazardous than the existing standard Class 3 commodity with plenty of air space among the loosely arranged paper cups. Similar judgment may also apply to the Class 4 commodity. This particular issue is the subject of an ongoing investigation. 
Since the protection recommendations in the DS include interpretations and extrapolations of full-scale sprinkler test data, it is useful to further examine the validity of the calculated SDF values by comparing them directly to the sprinkler test results. This comparison is shown in Table 1 . Note that the SDF values are computed for a $4.6 \mathrm{~m}$ fuel array in a $7.6 \mathrm{~m}$ building, while the full-scale tests were conducted for a 6.1 $\mathrm{m}$ fuel array in a $9.1 \mathrm{~m}$ building. Therefore, a reasonable comparison between the two should be carried out by reducing the full-scale test results approximately by $20 \%$. By the use of this approximation, it is clear that the estimated SDF values are within the range of the sprinkler test results except for Class 3 . The exception is again attributed to the limited test data for the Class 3 commodity and the fact that a very different commodity (densely packed paper cards in corrugated box) was used in the full-scale sprinkler test from the standard Class 3 commodity (loosely packed paper cups in corrugated boxes).

From the comparisons shown in Fig. 9. and Table 1., it appears that the SDF-based classification reasonably represents the fire hazard levels of the commodities of interests. However, before concluding that commodity classification can be determined based on the SDF values, two questions are worth examining: (1) will the ranking of CDF change with different fuel array heights? and (2) are there qualitative differences among the tested commodities in terms of their responses to water suppression? These questions can be answered by the use of suppression correlations developed from commodity classification test results.

Table 1. Comparison of SDF and Sprinkler Test Results.

\begin{tabular}{ccc}
\hline Commodity & Estimated SDF $(\mathrm{mm} / \mathrm{min})$ & Full-Scale Test $(\mathrm{mm} / \mathrm{min})$ \\
\hline Class 2 & 6.9 & $0.00 \sim 15.1$ \\
Class 3 & 19.9 & $12.2 \sim 17.9$ \\
Class 4 & 19.9 & $17.9 \sim 32.6$ \\
SPC & 25.6 & $24.4 \sim 32.6$ \\
Cartoned Meat Trays & 26.9 & $24.4 \sim 32.6$ \\
\hline
\end{tabular}

\section{Suppression Correlations}

Suppression correlations for all tested commodities were developed using the technical approach discussed earlier. Two examples are shown in Fig. 10. with comparisons to experimental data. Note that the scales in Fig. 10. are very different for the plastic pallets from those for SPC due to the small damage fractions for the exposed plastic commodities. In cases where the fire is out of control after water application, the damage fraction is deemed to be approximately unity and thus beyond the scope of the suppression correlations. Such data points are not included in Fig. 10.

The fact that different scales are needed for commodities in corrugated boxes and exposed plastic commodities itself reveals the difference between the two. For commodities in corrugated boxes, the damage fractions can be as large as $20-40 \%$ when the fire is still controlled. On contrast, the damage fractions for the exposed plastics have to be well below $10 \%$ to achieve the minimum level of control of fire spread. This difference has prompted further investigation of the response to water suppression by these two types of commodities.

In order to better describe the behaviors of commodities in fire suppression, the suppression correlation given in Eq. 3. is recast into a new form of

$\Delta Q_{e x} / \Delta Q_{0}=a\left(\dot{Q}_{w}^{*}\right)^{b}$,

where the cooling parameter $\dot{Q}_{w}^{*}$ is defined as $\dot{Q}_{w}^{*}=\left(\dot{Q}_{w} / \dot{Q}_{b e}\right)\left(\dot{Q}_{b e} / \dot{Q}_{\max }\right)^{c / b} n^{e / b}$, standing for the overall cooling power relative to the burning intensity at the beginning of extinguishment. When the experimental data are plotted in this form, the differences in suppression behaviors for the two types of commodities mentioned above become evident. Two examples are given in Fig. 11. The damage fractions of the exposed plastics (plastic pallets and exposed meat trays) vary only in a narrow range; beyond the upper limit of the range, the fire goes immediately out of control with damage fractions reaching unity. On contrast, the damage fractions for commodities stored in corrugated boxes increase in a much more gradual manner with decreasing cooling parameter. As a result, it is more difficult to achieve successful protection for the exposed plastic commodities than their counterparts stored in corrugated boxes, even if the two require the 
same water flux. Practically, this implies that early suppression fast response type of sprinkler protection should be recommended to protect the exposed plastics, while control mode sprinklers can be used to protect the commodities stored in corrugated boxes.
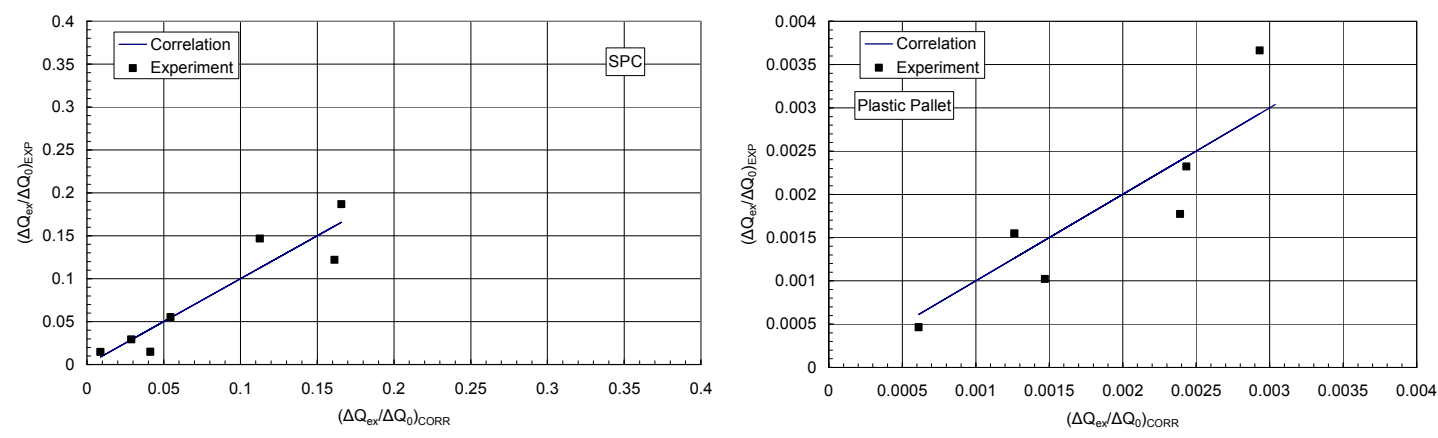

Fig. 10. Suppression correlation of tested commodities.
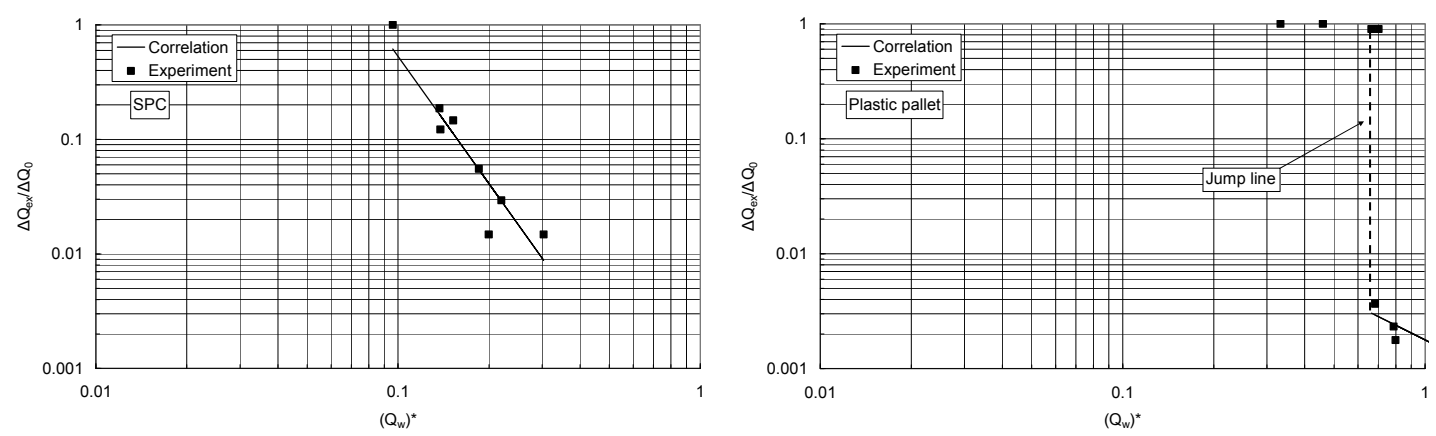

Fig. 11. Responses to water suppression by different commodities.

Once the suppression correlations are developed, estimation of CDF becomes possible for different fuel array heights. It is assumed in this work that for the same sprinkler condition, the damage fraction is a constant, the quantities $\dot{Q}_{\max }$ and $\Delta Q_{0}$ are proportional to the fuel array height and the value of $\dot{Q}_{b e}$ for $n$ tier fuel arrays can be estimated by holding $\left(\dot{Q}_{b e}\right)_{n-\text { tier }} / n$ invariant. It is recognized that the last assumption is crude because the HRR at the beginning of extinguishment should be determined by the HRR time history and the sprinkler condition. However, prediction of fire growth based on fuel properties and first principles is still not possible at this time. As a result, the assumptions made above are deemed adequate for the purpose of the present work. The variability of the predicted SDF using different $\dot{Q}_{b e}$ assumptions is also examined, e.g. simply keeping $\dot{Q}_{b e}$ constant or doubling $\dot{Q}_{b e}$ from 3- to 6-tier fuel array. The results show no significant impact on the ranking compared to that based on the aforementioned assumption.
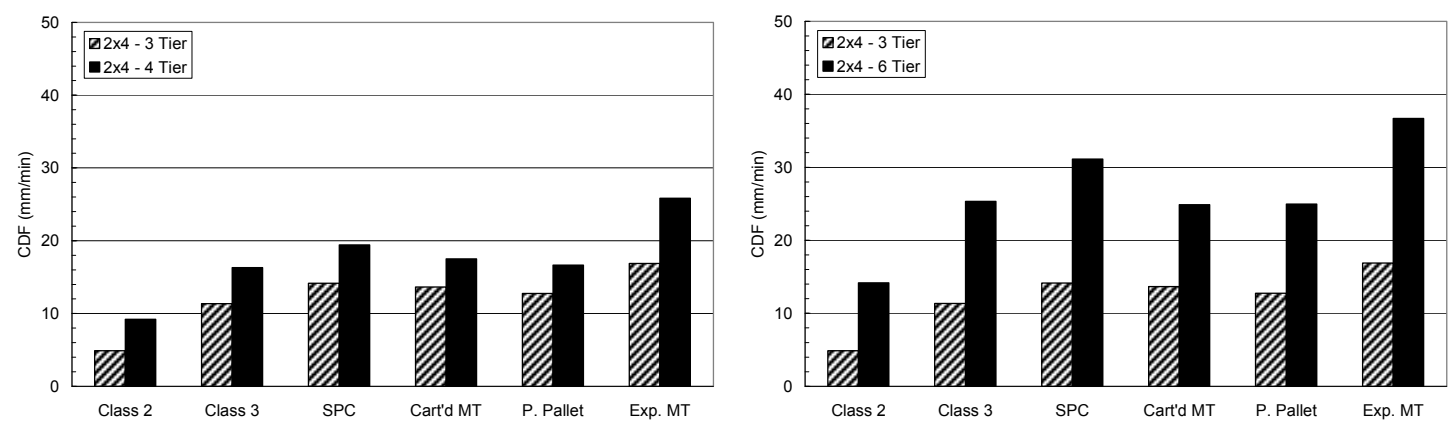

Fig. 12. Comparison of estimated CDF values for different fuel array heights. 
Comparisons of the CDF values for various fuel array heights are given in Fig. 12., which shows clearly that the ranking of measured CDFs from 3-tier fuel arrays is also valid for higher fuel arrays. Since the change of $\dot{Q}_{b e}$ from one fuel array height to another for a fixed sprinkler condition is not significant, the result shown in Fig. 12. essentially confirmed the validity of projecting the CDF to the SDF and ranking the commodities based on the measurements obtained from 3-tier high fuel arrays.

\section{SUMMARY AND CONCLUSIONS}

In order to assess the classification of commodities for sprinkler protection, fifty-six suppression tests were conducted using representative fuels, including Class $2-4$, SPC, cartoned meat trays, plastic pallets and exposed meat trays. The measured HRRs and applied water fluxes were used to calculate the CDF and develop suppression correlations for each tested commodity. The CDF values were then introduced into an ADD correlation to compute the critical SDF values, which were compared to the protection recommendations in existing FM Global Data Sheet and full-scale sprinkler test results to validate the SDFbased ranking of fire hazards for sprinkler protection. In addition, the responses of various commodities to water suppression and the effect of fuel array heights were examined by the use of suppression correlations.

The HRR measurements together with the CDF calculation procedure show that the CDF values can be obtained within an uncertainty of $\pm 3 \mathrm{~mm} / \mathrm{min}$. Examination of the CDF values using the suppression correlations suggests that the ranking remains the same for various fuel array heights. Furthermore, the suppression correlations also show that the responses to water suppression for the exposed plastic commodities appear to be very different from those for the cartoned commodities. As a result, these two types of commodities should be treated separately.

Once the CDF are determined, the ADD correlation can be used to project the SDF values. Comparison of the SDF values and the existing DS as well as full-scale sprinkler test results shows that the SDF is a reasonable choice for ranking either cartoned commodities or exposed plastic commodities. The relatively large differences in the comparison for Class 3 and 4 commodities might be attributed to the fact that loosely packed fuels are used in the present work, which may not be sufficiently representative for these types of commodities in the real world. This issue is currently subject to an ongoing research and may lead to the development of new representative commodities in the future.

\section{ACKNOWLEDGEMENT}

The authors are grateful to Drs. Robert G. Bill Jr., Louis A. Gritzo, John L. de Ris, Hong-Zeng Yu and Christopher Wieczorek for their invaluable discussions and encouragement on this work, and Ms. Kristin Jamison for conducting part of the experimental work. In addition, all tests were carried out with the help of the staff on FM Global Research Campus, which is truly appreciated.

\section{REFERENCES}

[1] Chicarello, P.J. and Troup J.M.A., "Fire Products Collector Test Procedure for Determining the Commodity Classification of Ordinary Combustible Products,” FM Global Technical Report J.I. OR0E5.RR, Norwood, MA, 1990, 61 p.

[2] Wu, P.K., 2005. Heat Flux Pipe in Large-Scale Fire Tests. Fire Safety Science 8: 1413-1424. doi:10.3801/IAFSS.FSS.8-1413

[3] Chan, T.S. and Kung, H.C., 2003. Comparison of Actual Delivered Density and Fire Suppression Effectiveness of Standard and Conventional Sprinklers in Rack-Storage Fires. Fire Safety Science 7: 445-456. doi:10.3801/IAFSS.FSS.7-445

[4] Yu, H.Z., Lee, J., and Kung, H.C., 1994. Suppression of Rack-Storage Fires by Water. Fire Safety Science 4: 901-912. doi:10.3801/IAFSS.FSS.4-901

[5] NFPA 13, "Standard for the Installation of Sprinkler Systems," 2007 Edition, National Fire Protection Association, Quincy, MA 02269, 2007.

[6] FM Global Property Loss Prevention Data Sheets 8-1 “Commodity Classification” May 2004. 
[7] Kung, H.C., Yu, H.Z., and Spaulding, R.D., 1986. Ceiling Flows of Growing Rack Storage Fires, Proceedings of the Combustion Institute 21: 121-128. doi:10.1016/S0082-0784(88)80238-8 